\title{
Look again
}

\section{Don Anderson argues that there is abundant evidence against the plume hypothesis, for an objective eye to consider.}

$\mathrm{I}$ astronomy and physics it is common to challenge and test the major reigning paradigms, including venerable ones such as the Big Bang, expansion, inflation, superstrings, dark matter and even relativity. In Earth sciences it is more common to hang on to cherished beliefs, such as continental and hotspot fixity, long after they have ceased to have any predictive power, and to rationalize discrepant observations by ad hoc modifications. The plate-tectonic revolution was strongly resisted but was so successful that the plume model, which followed on its heels, largely escaped criticism. Plumes were devised to overcome certain perceived shortcomings of plate tectonics such as the existence of volcanic chains and continental flood basalt provinces. Despite major shortcomings, the plume idea has been accepted and modified but seldom challenged or tested. Alternatives are often scoffed at rather than being seriously considered. Plumes have become unquestioned dogma rather than a testable idea.

The author and editors are to be complimented for publishing an alternate view for one of the classic textbook hotspots. The plume suggestion of Morgan (1971) and Wilson (1963) attempted to explain long-lived melting anomalies such as Iceland, Yellowstone, Hawaii and about 15 other volcanic provinces in terms of narrow, hot, stationary plumes, jets or thermals from the core-mantle boundary. The list later grew to about 170, the number of volcanic features that for various reasons were not considered plate boundary or incipient boundary features. The original speculation was an elegant idea and gave several specific testable predictions about heat flow, magma volume, fixity, and parallelism of island chains. One prediction was that there had to be about 20 plumes equivalent to Hawaii and these represented narrow upwellings compensated by distributed diffuse downwellings. Geophysical measurements including mantle tomography have shown that these predictions were wrong. The large predicted plume heads, easy to spot in tomographic images (Anderson et al. 1992) and uplift data, were not there. Alternative ideas involving crack propagation, mantle heterogeneity and small-scale convection must now be considered. The most serious observational problem with the plume idea is the lack of any evidence for high magma temperatures or high heat flow around hotspots or for thermal uplift (Anderson 1999, 2000). Athermal mechanisms such as magma focusing, magma fracture and corner flow must be entertained to explain regions of excess magmatism without uplift, high magma temperatures or high heat flow. Plate tectonics itself introduces thermal and chemical heterogeneity into the mantle so some regions will have greater or lesser amounts of fusable material and melting as a result.

The plume hypothesis has proven resistant to falsifications because rationalizations have been adopted for all discrepant data. It was fixity that convinced most workers that plumes were more appropriate than crack or stress-based hypotheses, but now we are told that fixity is not expected and is, on the contrary, an argument in support of plumes. Other rationalizations include large radius of influence, large distance lateral flow and explanations for the absence of uplift, heat flow and expected geochemical anomalies. Instabilities originating at the $650 \mathrm{~km}$ phase boundary have been proposed, even though this is a mineralogical phase transition and not a thermal or chemical boundary and such shallow plumes do not have the strength to do what they were originally proposed to do, such as breaking up continents, keeping ridges open and providing massive amounts of basalt through thick lithosphere. Finally, the pick-and-choose technique has been used to cull the official hotspot list down to between 7 and 10 as detailed studies eliminate plumes as credible explanations for the data. This leaves most "hotspots" unexplained. It is unlikely that Foulger's important observations will change many minds. Persuasive evidence against the plume hypothesis and accessible deep mantle reservoirs has been available for decades. The most serious problems underlying the plume hypothesis involve unrealistic assumptions about the physics and thermodynamics, the normal background temperature, melting temperature and homogeneity of the upper mantle. In plume calculations the upper mantle is unrealistically assumed to be cold, dry and subsolidus and more or less isothermal at a given depth. Large volumes of magma are assumed to reflect locally elevated temperatures imported from great depth, rather than differences in fertility, upper mantle temperature, melting point or focusing. The average mantle potential temperature is more likely closer to $1350{ }^{\circ} \mathrm{C}$ than to $1200{ }^{\circ} \mathrm{C}$ and the melting point is likely to be lower than dry pyrolite (Korenaga and Kelemen 2000, Anderson 2000). This makes an enormous difference. If normal upper mantle is mainly close to or above the solidus, plus or minus normal fluctuations, then the plume hypothesis is unnecessary. The asthenosphere has low viscosity and can flow towards regions of thin lithosphere without a plume. The long-distance lateral transport of plumehead material recently proposed (Sleep 1997) is an ad hoc adjustment to the deep plume hypothesis and brings it closer to alternative views regarding shallow distributed sources of heat and magma. Plumes are point sources of pollution and require large lateral transport to service the widespread volcanism attributed to them. A partially molten asthenosphere provides a widespread and readily available source of magma, needing only lithospheric extension to localize magmatism. Asthenospheric material and chemical heterogeneity need not originate at the core-mantle boundary or any deep thermal boundary layer.

The basic geochemical assumption behind plumes is that of a chemically homogeneous upper mantle. It is assumed that if mid-ocean ridge basalts come from the upper mantle then chemically different basalts must come from the lower mantle. This is not only a logical fallacy but is likely to be false (Anderson 1989, 1999). Sampling theory and the central limit theorem, however, show that large volume integrators such as oceanic ridges should be more homogenous and should exhibit less extreme values than smaller scale samplers such as oceanic islands. Thus, it is to be expected that ocean island basalts should be more geochemically diverse than mid-ocean ridge basalts, but have 
a similar mean. This is borne out by observation - many seamounts and oceanic islands have average isotopic ratios similar to ridge basalts, but greater variance. In the presence of mantle inhomogeneity, it is thus unnecessary to invoke a separate, isolated yet accessible, oceanisland basalt "reservoir" (Anderson 2001b).

Many fluid dynamic plume simulations adopt the so-called Boussinesq approximation, Cartesian geometry and large core heat flows, meaning there is a symmetry between the upper and lower thermal boundary layers. Great pressure suppresses thermal expansion and the local Rayleigh number so thermal upwellings in the deep mantle are large, weak, sluggish and longlived (Anderson 2001a). Most of the buoyancy, heat flow, conductive cooling and radioactive heating are concentrated in the outer layers of the Earth. The result is plate tectonics. The active surface boundary layer and associated mantle convection certainly overwhelm contributions from any deep thermal boundary layers which must be weaker. The fundamental physics of Earth is much more consistent with plate tectonics, mantle convection and magmatism being driven from the surface, and not by the deep interior. Plate tectonic forces not only drive the plates but can also break them, as can buoyant magma from below. This is an alternative to so-called hotspot tracks. This option is not available if the plates are rigid and permanent and the shallow mantle is isothermal and well below the melting point, assumptions of the plume hypothesis (Anderson 2001b).

It is the physics and the invalid assumptions, as much as observations, that make the plume hypothesis untenable. A more consistent hypothesis will have lithospheric and stress components and a heterogeneous, non-isothermal mantle, as expected from plate tectonics, and recycling of crust and lithosphere. When pressure is correctly taken into account, it is likely that mantle dynamics will prove to be a top-down system, organized by the tectonic plates and cooling lithosphere, rather than by plumes and core heat (Anderson 2001a). Magmatism, both at current and incipient plate boundaries, is a natural result of plate tectonics on an Earth-sized planet with a warm volatile-rich interior and a thin outer shell.

References

Anderson D L 1999 in James Hutton - Present and Future Craig GY and Hull J H (eds) Spec. Pub. of the Geol. Soc. London 150 13-35. Anderson D L 2001a Science 293 2016-18.

Anderson D L 2001b Earth Planet. Sci. Lett. 193 77-82.

Anderson D L 2000 Geophys. Res. Lett. 27 3623-26.

Anderson D L, Tanimoto T and Zhang Y-S 1992 Science 256 1645-50.

Anderson D L 1989 Theory of the Earth Blackwell Scientific

Publications, Boston, 366

Korenaga J and P Kelemen 2000 Earth Planet. Sci. Lett. 184

251-68.

Morgan W 1971 Nature 23 42-43.

Sleep N H 1997 J. Geophys. Res. 102 10001-12.

Wilson J T 1963 Can. J. Phys. 41 863-70. 